\title{
Carbon Nano Tube Interconnects for Hardware Architectures and Inter-Chip Connections -A Simulation Based Analysis
}

\author{
Jaisimha Manipatruni, Sohana Domathoty \\ Centre for Nanotechnology, Department of Computer Science \& Systems Engineering \\ Andhra University College of Engineering/Visakhapatnam, India.
}

Abstract

This work proposes a method of application of Carbon Nano Tube (CNT) Structures for Chip Interconnections in place of conventional Copper/Aluminium Chip Interconnects for efficient and High-Performance Computing Hardware Systems. Simulations are made for analysing Current carrying capacity and Number of Channels present per area of a Multi Walled Carbon Nano Tubes Bundle of 7 nm CNT size. Large conduction channels were observed at room temperature conditions and can be exploited for faster Interconnect speed thereby reducing the Process Latency and Electromigration

Keywords: Carbon Nanotubes, Interconnects, Conduction Channels, CNT Bundles

\section{Introduction}

Conventionally the design of hardware is done by using standard VLSI and Embedded System design rules which use conventional materials for both Chip Design and Interconnects for the most part. With advent of technologies like IOT ,5G and Artificial Intelligence which demand High Performance Computing and Low Latency it is almost inevitable to introduce Non-

Conventional Materials for Computer Systems Design by which we can achieve better and efficient End to End Experience. With advancement of Nanotechnology and Microelectronics we are able to push the Moore's Law further by reducing the size of Chip Components and attaining better compute. Latency is one bothering challenge that Computer Architectures deal with since a very minute delay in transfer of Data or Signal can lead to Hardware Hazards, Stagnation of processes and Pipe Line Collapses in worst cases. In order to cope up with Latency challenges there is a need for application of Non-Conventional Materials for Chip Design and Fabrications.

Carbon Nano Tubes are promising alternatives to Copper/Aluminium based electronic design materials as we see CNTs have extraordinary properties which can be tailored according to the application and use cases.

We observe a large number of Conduction Channels and excellent Current Carrying Capacity in Multi Walled Carbon Nano Tubes on Simulations done on CNT Analyzer Tool Box Software. On proper simulations for various conditions well framed designs can be laid out for development and fabrication of Interconnects completely or partially replacing the conventional interconnects.

2.Interconnects:
Conventionally Copper based interconnects are used for VLSI and Architecture design. As interconnect feature sizes shrink, copper electrical resistance will increase due to surface and grain boundary scatterings and additionally surface roughness.

Moreover, wires, particularly power and ground lines, have risk of electromigration increase in current densities

The resistance of copper interconnects, with crosssectional dimensions of the order of the mean free path of electrons ( $40 \mathrm{~nm}$ in metal at space temperature) in current and close technologies, is increasing speedily beneath the combined effects of increased grain boundary scattering, surface scattering and also the presence of the extremely resistive diffusion barrier layer

Interconnects play a very crucial role in Architectural design of hardware and it is reported that majority of Hardware Hazards are due to latent interconnects. In order to overcome this the designers have adopted several micro and nano composites for interconnects.

\begin{tabular}{lcc}
\hline \hline \multicolumn{1}{|c}{ Metal } & Melting point $\left({ }^{\circ} \mathrm{C}\right)$ & Resistivity $(\rho)$ at $298 \mathrm{~K}(\mu \Omega-\mathrm{cm})$ \\
\hline Silver (Ag) & 961 & 1.587 \\
\hline Copper (Cu) & 1084 & 1.678 \\
\hline Gold (Au) & 1064 & 2.214 \\
\hline Aluminum (Al) & 660 & 2.650 \\
\hline Tungston (W) & 3422 & 5.280 \\
\hline \hline
\end{tabular}




\section{Limitations of Copper Interconnects}

The existing Copper technology for VLSI interconnects can't be scaled further on the far side $22 \mathrm{~nm}$ because the grain boundary scattering of electrons happens and also the interconnects square measure liable to electromigration. Further, an extra lining is required for $\mathrm{Cu}$ vias to avoid diffusion into chemical element at high temperatures that makes the technology unattractive. Moreover, the steadiness of the Copper wires at high currents and the signal strength at terribly high frequencies of the order of tens of Giga $\mathrm{Hz}$ is of great concern. Carbon nanotubes are getting high popularity and are promising interconnect material

Electromigration is one of the biggest problems the Semiconductor design usually face. Electromigration refers to removal of metal surface from due to high density current passing through the interconnects. Initially Aluminium interconnects were replaced with copper interconnects to overcome this problem of Electromigration (EM), nevertheless EM in Copper is still a limitation for High Current Densities

These are some limitations which can be overcome by using CNT as interconnect material due to Low latency in CNTs and High Current Carrying Capacity of CNTs both as bundles and individuals.

Thus we can come to an initial observation that CNTs are one of the advancing and promising interconnect materials for VLSI design and Computer Hardware Architectures on a Chip and Physical Design Level.

\section{Simulations and Results}

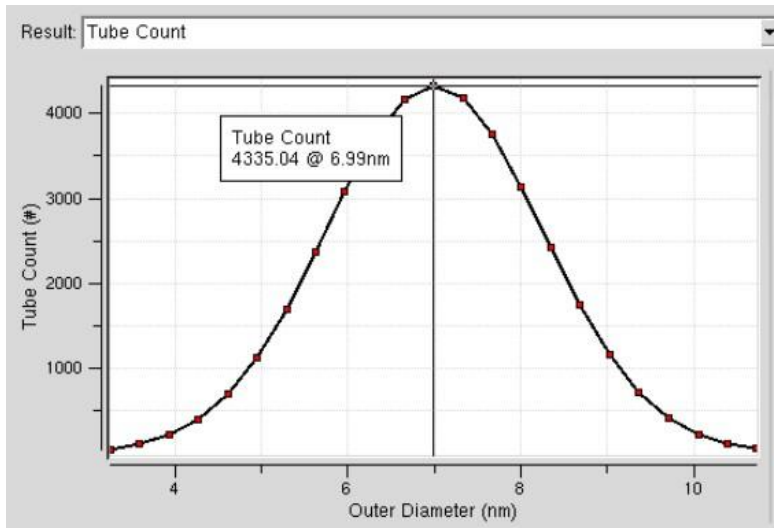

Fig2: Tube Count at $6.99 \mathrm{~nm}$ CNT Bundle

From Fig,2 The Tube count of the CNT Bundle is obtained. For a CNT Bundle of $7 \mathrm{~nm}$ the maximum number of Tubes were observed at $6.99 \mathrm{~nm}$. This gives evidence that CNT bundles of much smaller diameters can be used for Interconnects due to large number of Tubes present for smaller diameters thereby reducing the size of interconnects without compromising number of carrying channels.
The simulations are performed on Carbon Nano Tube Bundles using Software techniques provided by Tool Box Software -Carbon Nano Tube Interconnect Analyser (CNTIA)V1.13 built by College of Nanoscale Science and Engineering, University of Albany, NY, USA.

Simulations are performed to obtain Number of Conduction Channels and Number of Nanotubes for Geometry of 7nanometer CNT Bundle at 300K Temperature.

\subsection{Simulation Parameters :}

\begin{tabular}{|l|l|l|l|}
\hline $\begin{array}{l}\text { CNT } \\
\text { Width }\end{array}$ & $\begin{array}{l}\text { CNT } \\
\text { Height }\end{array}$ & $\begin{array}{l}\text { CNT } \\
\text { Length }\end{array}$ & $\begin{array}{l}\text { Average } \\
\text { Diameter }\end{array}$ \\
\hline $2 \mathrm{~nm}$ & $2 \mathrm{~nm}$ & $4 \mathrm{~nm}$ & $7 \mathrm{~nm}$ \\
\hline
\end{tabular}

Geometries

Table1:Simulation

\subsection{Output Log}

\begin{tabular}{|l|l|}
\hline $\begin{array}{l}\text { Number of Conduction } \\
\text { Channels }\end{array}$ & $6.472955 \mathrm{e}+02$ \\
\hline Inductance & $5.101597 \mathrm{e}-12$ Henry \\
\hline Capacitance & $4.444079 \mathrm{e}-17$ Faraday \\
\hline Resistance & $1.343828 \mathrm{e}-02 \mathrm{Ohm}$ \\
\hline Number of CNTs/Bundle & 39883 \\
\hline Drift Velocity & $2.656538 \mathrm{e}+07 \mathrm{~m} / \mathrm{s}$ \\
\hline Temperature & $300 \mathrm{~K}$ \\
\hline Propagation Delay & $4.139530 \mathrm{e}-07 \mathrm{ps}$ \\
\hline
\end{tabular}

Simulations

\subsection{Electrical Conduction Channels}

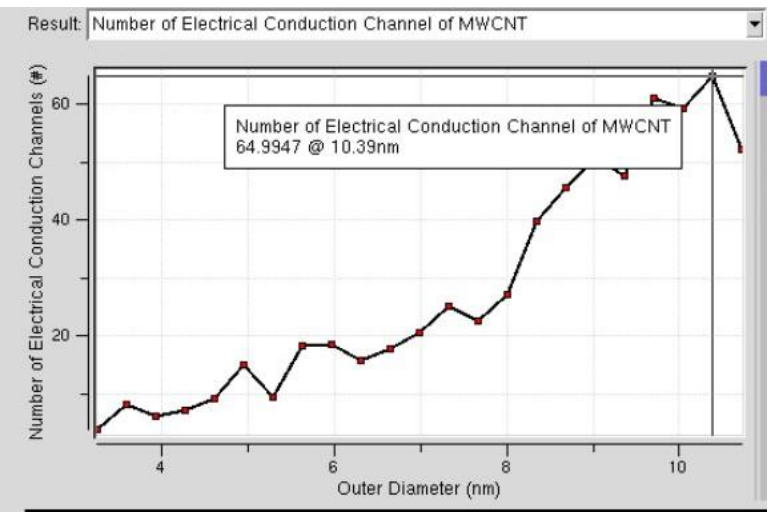

Figure 3: Number of Electrical Conduction Channels of MWCNT Bundle

From Fig3, It has been observed that we can obtain 64.997 Number of Conduction Channels at 10nm Outer Diameter which is Optimal for this Simulation Profile. Although the Optimal value varies depending upon the Geometry of Individual CNTs and Bundle. 
The number of Conduction channels obtained for a very minute diameter is significant for CNTs. This can be considered as one special character of CNT which makes it a promising alternate to conventional materials and by which we can reduce the latency interconnects. Since the current carrying capacity is high and the presence of large number of Conducting Channels for smaller diameters solves the challenges of Electromigration.

\section{Conclusion and Future Scope}

The simulations have been performed on CNT Bundles and a preliminary conclusion has been made that CNTs by virtue of their excellent electronic properties like Number of Channels per Diameter and Number of Tubes per Bundle, these can be used extensively in combination with existing Copper and Conventional Interconnects thereby overcoming the Physical Design Challenges Namely Latency and Electromigration.

CNTs are undoubtedly modern interconnect materials which can overcome the limitations of existing interconnect materials. Future work includes synthesis of High Yield CNTs, Floor Planning of Circuit Design and fabrication into a usable hardware component.

Integration of CNTs into VLSI and Micro-electronics can preserve the Moore's Law and gives a possibility of High-Performance Computing Systems like Photonic Computing, Nano Integrations and Spin-Based Memory devices.

\section{References}

[1] Thompson SE, Parthasarathy S. Moore's law: The future of Si microelectronics. Materials

Today. 2006;9:20-25

[2] Grill A, Gates SM, Ryan TE, Nguyen SV, Priyadarshini D. Progress in the development and understanding of advanced low $\mathrm{k}$ and ultralow $\mathrm{k}$ dielectrics for very large-scale integrated interconnects - State of the art. Applied Physics Reviews. 2014;1:011306-011312

[3] Bohr M. MOS transistors: Scaling and performance trends. Semiconductor International.

1995;18(6):75-80

[4] Grill A. Porous pSiCOH ultralow-k dielectrics for chip interconnects prepared by

PECVD. Annual Review of Materials Research. 2009;39:49-69

[5] Kim CY, Navamathavan R, Lee HS, Woo JK, Hyun MT, Lee KM, Jeung WY, Choi CK.

Ultraviolet irradiation effect on the properties of leakage current current and dielectric

breakdown of low-dielectric-constant $\operatorname{SiOC}(\mathrm{H})$ films using comb capacitor structure.

Thin Solid Films. 2011;519:6732-6736

[6] Broussous L, Berthout G, Rebiscoul D, Rouessac V, Ayral A. Mechanical properties of a plasma-modified porous low-k material. Microelectronic Engineering. 2010;87:466-469

[7] Cheng YL, Wang YL, Liu CW, Wu YL, Lo KY, Liu CP, Lan JK. Characterization and reliability of low dielectric constant fluorosilicate glass and silicon rich oxide process for deep sub-micro device application. Thin Solid Films. 2001;398-399:544-548 